\title{
Geometric-phase backaction in a mesoscopic qubit-oscillator system
}

\author{
G. Vacanti, ${ }^{1}$ R. Fazio, ${ }^{1,2}$ M. S. Kim,${ }^{3}$ G. M. Palma, ${ }^{4}$ M. Paternostro, ${ }^{5, *}$ and V. Vedral ${ }^{1,6,7}$ \\ ${ }^{1}$ Center for Quantum Technologies, National University of Singapore, 1 Science Drive 2, Singapore \\ ${ }^{2}$ NEST, Scuola Normale Superiore \& Istituto di Nanoscienze-CNR, I-56126 Pisa, Italy \\ ${ }^{3}$ QOLS, Blackett Laboratory, Imperial College London, SW7 2BW, United Kingdom \\ ${ }^{4}$ NEST Istituto Nanoscienze-CNR and Dipartimento di Fisica, Univerisita' degli Studi di Palermo, Via Archirafi 36, I-90123 Palermo, Italy \\ ${ }^{5}$ Centre for Theoretical Atomic, Molecular, and Optical Physics, School of Mathematics and Physics, Queen's University, \\ Belfast BT7 INN, United Kingdom \\ ${ }^{6}$ Clarendon Laboratory, University of Oxford, Parks Road, Oxford OX1 3PU, United Kingdom \\ ${ }^{7}$ Department of Physics, National University of Singapore, 3 Science Drive 4, Singapore
}

(Received 16 August 2011; published 29 February 2012)

\begin{abstract}
We illustrate a reverse Von Neumann measurement scheme in which a geometric phase induced on a quantum harmonic oscillator is measured using a microscopic qubit as a probe. We show how such a phase, generated by a cyclic evolution in the phase space of the harmonic oscillator, can be kicked back on the qubit, which plays the role of a quantum interferometer. We also extend our study to finite-temperature dissipative Markovian dynamics and discuss potential implementations in micro- and nanomechanical devices coupled to an effective two-level system.
\end{abstract}

DOI: 10.1103/PhysRevA.85.022129

PACS number(s): 03.65.Vf, 42.50.Dv, 42.50.Pq, 85.85.+j

\section{INTRODUCTION}

Although it is commonly believed that quantum mechanical behaviors are preclusive characteristics of microscopic systems, various nonclassical effects have been theoretically predicted and experimentally observed in massive systems. In optomechanical devices [1-7] as well as in setups involving superconducting qubits coupled with nanomechanical resonators [8,9], a variety of quantum behaviors is observable even at high temperatures and in the presence of strong dissipative processes. In general, the problem of enforcing quantumness under unfavorable conditions has received great attention in recent years, being the topic of great interest both from a technological and from a fundamental point of view. In this line of thought, we consider a system in which genuine nonclassical features not only survive to temperature and dissipation, but are indeed induced by these environmental influences. While quantum effects like entanglement and negative values of Wigner function have been treated elsewhere [10], here we focus on the study of the generation and detection of a geometric phase $[11,12]$ in a harmonic oscillator. In the spirit of previous works [13], we reconsider the Von Neumann measurement scheme, which models the measurement process as a coupling between a large measurement apparatus, used as a probe, and the microscopic system on which the measurement is performed, under a "reverse" prospective, using the microscopic system (a qubit) to measure the geometric phase attached to the macroscopic one (a harmonic oscillator).

\section{DESCRIPTION OF THE MODEL}

To illustrate the idea we consider a general Hamiltonian model and defer the presentation of a physical scenario suited for its realization to the second part of this work. We thus take

\footnotetext{
*m.paternostro@qub.ac.uk
}

a two-level system with logical states $\{|0\rangle,|1\rangle\}$ (which we dub the control qubit) coupled with a harmonic oscillator through the interaction Hamiltonian

$$
\hat{H}=\hbar \eta|0\rangle\langle 0| \otimes\left(\hat{b}^{\dagger} e^{-i \varphi}+\hat{b} e^{i \varphi}\right),
$$

where $b$ and $b^{\dagger}$ are the annihilation and creation operators for the oscillator, $\eta$ is the coupling constant, and $\varphi$ is a phase that can be externally adjusted to change the direction of the field's quadrature in phase space. As long as $\eta$ and $\varphi$ are constant in time, the unitary operator describing the conditional time evolution of the system is

$$
\hat{U}_{\varphi}(t)=|1\rangle\langle 1|\otimes \hat{\mathbb{1}}+| 0\rangle\langle 0| \otimes \hat{D}[\zeta],
$$

where $\hat{D}[\zeta]=e^{\zeta \hat{b}^{\dagger}-\zeta^{*} \hat{b}}$, with $\zeta=-i \int_{0}^{t} \eta e^{-i \varphi}$, is a displacement operator acting on the oscillator. Equation (2) describes a spin-state-dependent displacement of the oscillator, which remains unperturbed if the control qubit is in $|1\rangle$ and is displaced by $\zeta$ if the control qubit is in $|0\rangle$. By changing $\varphi$ in time, we can drag the state of the harmonic oscillator along a nontrivial path in phase space. In particular, by varying $\varphi$ along a closed loop we can associate a purely geometric phase to the state of the harmonic oscillator. Following Refs. [14,15], we assume that the value of $\varphi$ is changed in $n$ time steps $\delta t$ such that $t=n \delta t, \delta \zeta_{i}=\dot{\zeta}_{i} \delta t$, and $\hat{U}(t)=\prod_{i=1}^{n} \hat{D}\left(\delta \zeta_{i}\right)$. Recalling that $\hat{D}(\alpha) \hat{D}(\beta)=\exp \left\{i \operatorname{Im}\left(\alpha \beta^{*}\right)\right\} \hat{D}(\alpha+\beta)$, we have

$$
\hat{U}(t)=\exp \left[i \operatorname{Im} \sum_{k=2}^{n} \delta \zeta_{k} \sum_{l=1}^{k-1} \delta \zeta_{l}^{*}\right] \hat{D}\left(\sum_{i=1}^{n} \delta \zeta_{i}\right) .
$$

In the continuous limit we take $\sum_{i=1}^{n} \delta \zeta_{i} \rightarrow \zeta, \sum_{k=2}^{n}$ $\delta \zeta_{k} \sum_{l=1}^{k-1} \delta \zeta_{l}^{*} \rightarrow \int \zeta^{*} d \zeta$ and assume that $\varphi$ is changed along a closed loop in a time $\tau$ such that $\zeta(\tau)=\zeta(0)$. Making use of Stokes' theorem [16], we find $\hat{U}(\tau)=e^{i \mathcal{A}} \hat{D}(0)$, with $\mathcal{A}$ the area enclosed by the cyclic path in parameter space [17].

Let us now suppose that the system can be initialized in the state $|\psi(0)\rangle=|+\rangle|\alpha\rangle$, where $|+\rangle=(1 / \sqrt{2})(|0\rangle+|1\rangle)$ is the state of the control qubit and $|\alpha\rangle$ is a coherent state of the 
(a)

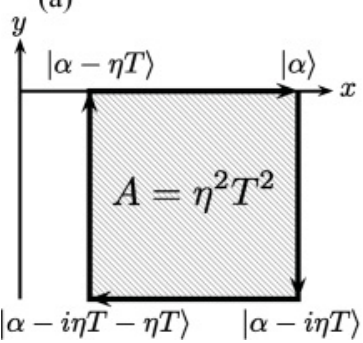

(b)

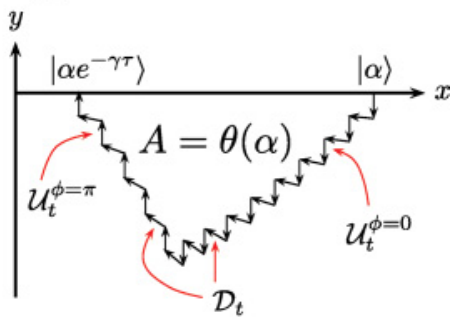

FIG. 1. (Color online) The oscillator's conditional dynamics pictured in phase space. (a) The oscillator is displaced along a square whose area is proportional to the phase $\theta$. (b) The oscillator is displaced while undergoing a dissipative process. Here $\hat{\mathcal{U}}_{\delta t}^{\varphi}$ and $\hat{\mathcal{D}}_{\delta t}$ are the superoperators describing the unitary and dissipative evolution of duration $\delta t$, respectively.

oscillator. We then change $\varphi(t)$ along a closed path spanned in a time $\tau$. The joint state of the qubit and oscillator at time $\tau$ will be

$$
|\psi(\tau)\rangle=(1 / \sqrt{2})\left(|1\rangle+e^{-i \mathcal{A}}|0\rangle\right) \otimes|\alpha\rangle .
$$

It should be remarked that Eq. (4) is exact and arises simply from the conditional oscillator evolution induced by its coupling to the two-level system as described in Eq. (1). To better understand this result, we discuss an illustrative example where we consider a rectangular path implemented by a stepwise change of $\varphi$ : its value is set to 0 for an interval of length $T$ and then changed to $\varphi_{n}=n \pi / 2$ at time $t=n T$ with $n=1,2,3$. As shown above, the state of the qubit acquires a phase $\vartheta=\mathcal{A}=\eta^{2} T^{2}$ equal to the area enclosed by the path [see Fig. 1(a)]. We now stress three important points. First, although in the above example the phase explicitly depends on $T$, it is invariant with respect to the parametrization of the path. Indeed, if the area enclosed by the path remains unchanged, even for an arbitrary dependence of $\zeta(t)$ on time, we would obtain the same result $\vartheta=\mathcal{A}$. Second, for a closed loop, $\vartheta$ does not depend on the amplitude $\alpha$ of the initial state of the harmonic oscillator. Third, the phase acquired by the harmonic oscillator is kicked back on the qubit state, which takes the role of a microscopic interferometer. As a final remark, it is important to stress that the geometric phase addressed here is a specific result of our interaction model and of the overall scheme that we have set up.

\section{THERMAL AND NONUNITARY CASE}

We now address the first two points mentioned above. As shown by Eq. (4), when the evolution of the system is purely unitary, the geometric phase picked up by the control qubit does not depend on the amplitude $\alpha$ of the oscillator's initial state. This leaves the value of $\theta$ unchanged even if the initial state of the oscillator is a thermal state $\rho_{V}=$ $\int d \alpha P(\alpha, 0, V)|\alpha\rangle\langle\alpha|$, where $P\left(\alpha, \alpha_{0}, V\right)=\frac{2}{\pi(V-1)} e^{-\frac{2\left|\alpha-\alpha_{0}\right|^{2}}{V-1}}$ is the Gaussian thermal distribution centered at point $\alpha_{0}$ in the phase space and having variance $V=\left(e^{\beta}+1\right) /\left(e^{\beta}-1\right)$, with $\beta=\hbar \omega_{m} / k_{b} \mathcal{T}\left(k_{b}\right.$ is the Boltzmann constant and $\mathcal{T}$ is the temperature of the oscillator). By taking the initial state $\rho_{0}=|+\rangle\langle+| \otimes \rho_{V}$, it is straightforward to see that a phase identical to the pure-state case is acquired by the qubit. In fact, the state at time $\tau$ reads $\rho(\tau)=|\varphi\rangle\langle\varphi| \otimes \rho_{V}$, where $|\varphi\rangle=\left(|1\rangle+e^{-i 2 \eta^{2} \tau^{2}}|0\rangle\right) / \sqrt{2}$. The invariance of $\theta$ in such a mixed-state scenario has also been confirmed using the framework for the evaluation of mixed-state geometric phases proposed in Ref. [19]. This shows that ignorance of the initial preparation of the state of the harmonic oscillator does not affect the possibility of generating and detecting a geometric phase.

We now assess the potential effects that nonunitary dynamics may have on the occurrence of the geometric phase under scrutiny. On one hand, the consideration of an explicitly open dynamics will make our proposal closer to the reality of the potential experimental situations that are addressed later in this work. On the other hand, it is reasonable to expect significant deviations from the results found so far when we are far from unitarity. We thus consider the oscillator to be affected by dissipation at rate $\gamma$ with the control qubit still evolving unitarily, a situation that is formally described by the master equation $\dot{\rho}=-(i / \hbar)[\hat{H}, \rho]+\hat{\mathcal{L}} \rho$, where $\hat{\mathcal{L}} \rho=\gamma\left(\hat{b} \rho \hat{b}^{\dagger}-\left\{\hat{b}^{\dagger} \hat{b}, \rho\right\} / 2\right)$ formally describes the oscillator's damping in a bath at zero temperature. Although we do not refer to any explicit experimental configuration, at this stage the analysis that we perform here agrees very well with the experimental observations on the open-system dynamics of mechanical systems available to date $[1,8]$. While the more realistic effects of a dissipative thermal bath are discussed later, this case is pedagogically quite useful, as it allows us to draw a particularly clear physical picture of the competition between the unitary displacement in phase space and the dissipative counteraction. In order to grasp this effectively, we divide the time window of the evolution in small intervals, each of length $\delta t$. Inspired by the Suzuki-Trotter formula [20], the dynamics can then be approximated by alternating a unitary evolution described by $\hat{\mathcal{U}}_{\delta t}^{\varphi} \rho=\hat{U}_{\varphi}(\delta t) \rho \hat{U}_{\varphi}^{\dagger}(\delta t)$ and the purely dissipative propagator $\hat{\mathcal{D}}_{\delta t} \rho=e^{\hat{\mathcal{L}} \delta t} \rho[21,22]$. After $N$ iterations, we have the evolved state

$$
\rho(N \delta t)=\left(\hat{\mathcal{D}}_{\delta t} \hat{\mathcal{U}}_{\delta t}^{\varphi}\right)^{N} \rho_{0} .
$$

This approach is particularly useful in analyzing a damped harmonic oscillator. Indeed, the action of the dissipative superoperator $\hat{\mathcal{D}}_{t}$ on the off-diagonal elements of a density matrix written in a coherent state basis is given by the dyadic expression [23]

$$
\hat{\mathcal{D}}_{t}\left|\lambda_{1}\right\rangle\left\langle\lambda_{2}\left|=\left\langle\lambda_{2} \mid \lambda_{1}\right\rangle^{1-\exp (-\gamma t)}\right| \lambda_{1} e^{-\gamma t}\right\rangle\left\langle\lambda_{2} e^{-\gamma t}\right|,
$$

where $\left|\lambda_{j}\right\rangle(j=1,2)$ are two coherent states. As we are interested in short-time intervals $\delta t$, we take $1-\exp (-\gamma \delta t) \simeq$ $\gamma \delta t$. Therefore, the action of $\hat{\mathcal{D}}_{\delta t}$ on the state of our system results in the displacement of the harmonic oscillator and the exponential decrease in its initial amplitude $\alpha$. Moreover, from Eq. (6) we see that a phase factor is attached to the off-diagonal elements of the density matrix. Such features are useful for closing the path across which the oscillator is displaced. To better stress the role played by dissipation, we consider a different unitary path in phase space with respect to the previous example. The path is such that the contribution to the geometric phase in the nondissipative regime is 0 . This allows us to clearly show how the dissipative process can induce a geometric phase on the system. Let us consider the 
case in which the phase $\varphi$ is set to 0 for $t \in\left[t_{0}, t_{0}+T_{1}\right]$ and to $\pi$ for $t \in\left[t_{0}+T_{1}, t_{0}+T_{1}+T_{2}\right]$. The state at the intermediate time $t_{0}+T_{1}$ is given by $\rho\left(t_{0}+T_{1}\right)=\left(\hat{\mathcal{D}}_{\delta t} \hat{\mathcal{U}}_{\delta t}^{0}\right)^{N_{1}} \rho_{0}$, with $N_{1} \delta t=T_{1}$. Taking the limit $\delta t \rightarrow 0$, this turns out to be

$$
\begin{aligned}
\rho\left(t_{0}+T_{1}\right)= & \frac{1}{2}\left(\left|1, \alpha_{1}\right\rangle\left\langle 1, \alpha_{1}|+| 0, \alpha_{1}-i \beta_{1}\right\rangle\left\langle 0, \alpha_{1}-i \beta_{1}\right|\right. \\
& \left.+e^{-i \theta_{1}(\alpha)} e^{-\Gamma_{1}}\left|0, \alpha_{1}-i \beta_{1}\right\rangle\left\langle 1, \alpha_{1}\right|+\text { H.c. }\right),
\end{aligned}
$$

where $\theta_{1}(\alpha)=\frac{\eta \alpha}{2 \gamma}\left(1-e^{-2 \gamma T_{1}}\right), \beta_{1}=\frac{\eta}{\gamma}\left(1-e^{-\gamma T_{1}}\right), \alpha_{1}=\alpha e^{-\gamma T_{1}}$, $\Gamma_{1}=\frac{\eta^{2}}{2 \gamma^{2}}\left[\gamma T_{1}+\frac{1}{2}\left(1-e^{-2 \gamma T_{1}}\right)-2\left(1-e^{-\gamma T_{1}}\right)\right]$. Equation (7) shows that the state of the oscillator at time $T_{1}$ is conditionally displaced by a quantity $-i \beta_{1}$. We can now proceed to evaluate the state of the system at time $t_{0}+T_{1}+T_{2}$ by setting $\varphi=$ $\pi$ and taking $\rho\left(t_{0}+T_{1}+T_{2}\right)=\left(\hat{\mathcal{D}}_{\delta t} \hat{\mathcal{U}}_{\delta t}^{\pi}\right)^{N_{2}} \rho\left(t_{0}+T_{1}\right)$, with $N_{2} \delta t=T_{2}$. This displaces the state of the oscillator by $i \beta_{2}$ in the opposite direction to what occurred at $T_{1}$. The time interval $T_{2}$ is chosen such that the oscillator displacement $-i \beta_{1}$ accumulated during $T_{1}$ is canceled [24]. By calling $\tilde{\tau}=T_{1}+$ $T_{2}$, the final state reads

$$
\begin{aligned}
\rho\left(t_{0}+\tilde{\tau}\right)= & \frac{1}{2}\left[\mathbb{1}+e^{-\Gamma}\left(e^{-i \theta(\alpha)}|0\rangle\langle 1|+\text { H.c. }\right)\right] \\
& \otimes\left|\alpha e^{-\gamma \tilde{\tau}}\right\rangle\left\langle\alpha e^{-\gamma \tilde{\tau}}\right|,
\end{aligned}
$$

where

$\theta(\alpha)=\eta \alpha \frac{1-2 e^{-\gamma T_{1}}+e^{-2 \gamma T_{1}}}{\gamma\left(2-e^{-\gamma T_{1}}\right)}, \quad \Gamma=\frac{\eta^{2}}{2 \gamma^{2}} \tilde{\Gamma}\left(\gamma, T_{1}, T_{2}\right)$,

with $\tilde{\Gamma}\left(\gamma, T_{1}, T_{2}\right)$ a dimensionless function that behaves as $\gamma^{3}$ for $\gamma \rightarrow 0$, thus ensuring that $\Gamma \rightarrow 0$ as $\gamma \rightarrow 0$ [25]. On the other hand, the phase $\theta(\alpha)$ goes to 0 when $\gamma \rightarrow 0$, as expected from our choice of the path in the dissipative case. It is easily seen that the phase $\theta(\alpha)$ gained by the oscillator in this process is equal to the area $\mathcal{A}$ enclosed by the displacement path in parameter space, as shown in Fig. 1(b). The detectability of this phase depends on the function $\Gamma$, which determine the decoherence rate for the off-diagonal terms in $\rho\left(t_{0}+\tilde{\tau}\right)$. Indeed, in order to achieve a nonvanishing phase, we should ensure that $\Gamma \ll 1$. Remarkably, while $\theta(\alpha)$ depends on the amplitude of the initial coherent state, $\Gamma$ does not. Surprisingly, by choosing $\eta / \gamma \ll 1$ (which embodies the weak-coupling condition between the oscillator and the control qubit), the requirement $\Gamma \ll 1$ is fulfilled. On the other hand, we can achieve any value of $\theta(\alpha)$ by making an appropriate choice for the value of $\alpha$, so as to compensate for the conditions required for a negligible damping.

This analysis can be extended to the case of a nonzerotemperature bath for which the dissipative part of the dynamics is accounted for by the Liouvillian $\hat{\mathcal{L}}_{T} \rho=\gamma(\bar{n}+1)\left(\hat{b} \rho \hat{b}^{\dagger}-\right.$ $\left.\left\{\hat{b}^{\dagger} \hat{b}, \rho\right\} / 2\right)+\gamma \bar{n}\left(\hat{b}^{\dagger} \rho \hat{b}-\left\{\hat{b} \hat{b}^{\dagger}, \rho\right\} / 2\right)$. A dynamical approach fully analogous to the dyadic-based one used above can be adopted, following the lines of Ref. [18]. In this case, the damping of a coherent state can be expressed in terms of displaced number states weighted by a thermal probability distribution determined by the actual value of $\bar{n}$. Yet the geometric phase can still be tracked for moderate temperatures, up to $0.1 \mathrm{mK}$, which marks the threshold above which thermal effects wash out the effect at hand, making the closure of a path in phase space basically impossible.

The approach described above can be applied so as to evaluate the effects on the geometric phase due to the thermal preparation of the state of the oscillator undergoing dissipative dynamics. We thus assume that the initial state of the oscillator is the displaced thermal state $\rho_{V}^{\alpha_{0}}=\int d \alpha P\left(\alpha, \alpha_{0}, V\right)|\alpha\rangle\langle\alpha|$. Following the lines sketched so far, we arrive at the evolved state

$$
\rho_{V}^{\alpha_{0}}\left(t_{0}+\tilde{\tau}\right)=\int d^{2} \alpha P\left(\alpha, \alpha_{0}, V\right) \rho\left(t_{0}+\tilde{\tau}\right),
$$

where $\rho\left(t_{0}+\tilde{\tau}\right)$ is given by Eq. (8). In light of the dependence of the phase $\theta$ on the amplitude $\alpha$ [as shown right after Eq. (8)], the control qubit and the oscillator end up in a correlated state. This complicates the calculation of the overall geometric phase associated with $\rho_{V}^{\alpha_{0}}\left(t_{0}+\tilde{\tau}\right)$. Nevertheless, it is still possible to evaluate the geometric phase by adopting the framework developed in Ref. [19], which is based on the probability that a measurement over the state of the control qubit has outcomes $\{|+\rangle,|-\rangle\}$ (with $| \pm\rangle=(|0\rangle \pm|1\rangle) / \sqrt{2})$. In order to understand this, let us first consider the state in Eq. (8) and project the control qubit onto the $\{| \pm\rangle\}$ basis. The corresponding outcome probabilities are given by $\mathcal{P}_{ \pm}=\left\langle \pm\left|\operatorname{Tr}_{m}\left\{\rho\left(t_{0}+\tilde{\tau}\right)\right\}\right| \pm\right\rangle$, where $\operatorname{Tr}_{m}$ denotes the partial trace over the oscillator's degrees of freedom. A straightforward calculation shows that $\mathcal{P}_{ \pm}=[1 \pm$ $v \cos \theta(\alpha)] / 2$, where $v=e^{-\Gamma}$. This reminds us of the fringes of an interferometer whose visibility is $v$ : the state of the composite qubit-oscillator system evolves along two branches (one associated with $|1\rangle$ and the other with $|0\rangle$ ), which can be seen as two arms of a Mach-Zehnder interferometer. The two components of the state of the system that have undergone the evolution ruled by $\hat{H}$ (and possibly the dissipative dynamics considered here) are then allowed to interfere by projecting the qubit state onto the superposed basis. This analysis offers us an operative interpretation of $\theta(\alpha)$. In fact, by changing it, the probabilities $\mathcal{P}_{ \pm}$change, reaching the complete inversion when $\theta(\alpha)=\pi$. Therefore, $\theta(\alpha)$ can be seen as the inversion of the outcome probabilities $\mathcal{P}_{ \pm}$. We can attach an analogous meaning to the phase associated with a mixed initial state. We thus now consider the state in Eq. (10), which gives us $\mathcal{P}_{ \pm}=(1 \pm|\Lambda| \cos [\tilde{\theta}]) / 2$ with $\tilde{\theta}=\arg (\Lambda)$ and

$$
\Lambda=v \int d^{2} \alpha P\left(\alpha, \alpha_{0}, V\right) e^{i \theta(\alpha)} .
$$

As in the case of a pure state, the phase $\tilde{\theta}$ is operatively defined through the inversion of the probabilities $\mathcal{P}_{ \pm}$. Figure 2(a) shows these quantities against the initial displacement $\alpha_{0}$ and the temperature $V$. Although the visibility of the fringes decreases with increasing temperature, it is possible to see a population inversion even for high values of $V$. Another very interesting situation is that in which the initial state is a nondisplaced thermal state; i.e., the Gaussian distribution in Eq. (10) is centered in $\zeta=0$. The behavior of the outcome probabilities $\mathcal{P}_{ \pm}$versus the thermal variance $V$ is shown in Fig. 2(b). A higher temperature results in an increase (decrease) in $\mathcal{P}_{-}\left(\mathcal{P}_{+}\right)$. The partial inversion of the probabilities is due to the average geometric phase $\tilde{\theta}(\alpha)$ picked up by the oscillator during the process.

\section{CONCLUSIONS}

In summary, we have shown how to generate a geometric phase on a system in which a qubit is coupled to a harmonic 

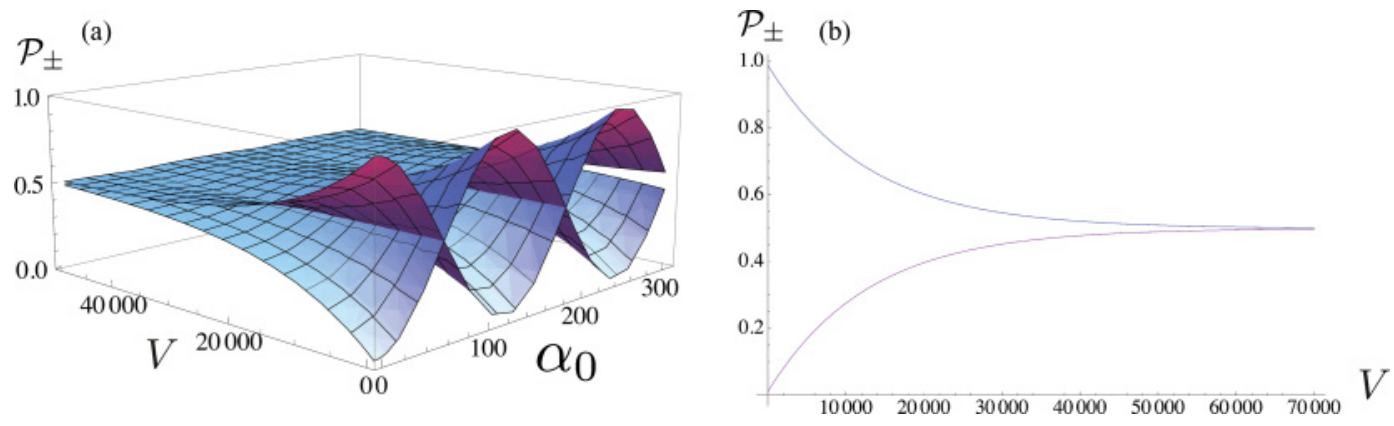

FIG. 2. (Color online) (a) Probabilities $\mathcal{P}_{+}$and $\mathcal{P}_{-}$versus the displacement $\alpha_{0}$ and the parameter $V$ for $\eta / \gamma=0.05$ and $\gamma T_{1}=20$. (b) The same probabilities versus $V$ for $\alpha_{0}=0$ and the same parameters as in (a).

oscillator. The phase can be detected using the qubit as an interferometer. We propose systems combining effective two-level devices to mechanical modes as potential scenarios for the implementation of our proposal [1,9]. In the nanoscale domain, Hamiltonian models of a form close to the one proposed here can be achieved by capacitatively combining a nanocantilever to a Cooper-pair box or growing a quantum dot on a nanobeam [9,26]. At the microscopic scale, on the other hand, the coupling in Eq. (1) can be engineered by means of a three-level atom trapped within the volume of a pumped optomechanical cavity field and off-resonantly coupled to the latter [10]. Analogous configurations have been proposed recently [27] as valid alternatives to consolidated schemes for the coupling between a mechanical mode and the vibrational degrees of freedom of a single atom, an ensemble of atoms, or a levitating nanoparticle [27,28]. Under the presence of a dissipative environment and for a mixed thermal state of the oscillator, the geometric phase can still be observed under conditions over the coupling between the qubit and the oscillator that can be matched experimentally $[1,8]$. For a nanobeam with a fundamental frequency of $\sim 100 \mathrm{MHz}$ coupled to a superconducting qubit at a rate $\eta \sim 1 \mathrm{MHz}$ and having a (realistic) decay rate of $\sim 1-10 \mathrm{MHz}$, which are values well within the validity of our approach, a temperature of $0.5 \mathrm{~K}$ keeps the probabilities $\mathcal{P}_{ \pm}$at the visible level by driving the mechanical mode with a two-tone signal [29], which is possible optically and electrically, thus covering both the micro- and the nanoscale configurations. In the first scenario, one could consider, for instance, a single Cs atom coupled to a light mechanical resonator (masses are typically in the nanogram range) in both the end mirror and the membrane-in-the-middle arrangements [1], as recently considered for the problem of coupling the external degrees of freedom of an atom to the vibrations of a massive mechanical oscillator [27]. High-finesse cavities with small waists are currently employed in controllable optomechanical experiments (finesse of $\sim 10^{5}$ with a waist of a few micrometers), thus guaranteeing a strong enough light-atom interaction that is suitable for the achievement of the effective Hamiltonian model proposed in Eq. (1). For the examples discussed here, all the experimental observations that are currently available are in full agreement with a Markovian description of the dynamics induced by the thermal background of phonons affecting the mechanical oscillator, thus making our study perfectly appropriate. Our proposal moves along the lines of an investigation assessing quantum effects in macroscopic systems. It enlarges the fan of indicators of quantumness at the mesoscale with a figure of merit, the geometric phase, that arises by virtue of the sole coherent qubit-oscillator interaction and survives against plainly adverse operating conditions.

Note added. Recently, a related article appeared reporting on the experimental generation of a Berry phase in the field of a planar microwave resonator coupled to a superconducting qubit [30]. The analysis presented there is fully in line with our proposal.

\section{ACKNOWLEDGMENTS}

M.P. and G.M.P. thank the CQT, National University of Singapore, where part of this work was done. We acknowledge financial support from the National Research Foundation and Ministry of Education in Singapore, the UK EPSRC, EUROTECH, EU projects GEOMDISS, QNEMS, and SOLID, the Royal Society, and the Wolfson Trust and College. M.P. thanks the UK EPSRC for financial support (Grant No. EP/G004759/1)
[1] M. Aspelmeyer, S. Groeblacher, K. Hammerer, and N. Kiesel, J. Opt. Soc. Am. B 27, A189 (2010); T. J. Kippenberg and K. J. Vahala, Science 321, 1172 (2008); F. Marquardt and S. M. Girvin, Physics 2, 40 (1993).

[2] S. Mancini, V. Giovannetti, D. Vitali, and P. Tombesi, Phys. Rev. Lett. 88, 120401 (2002).

[3] M. Paternostro, D. Vitali, S. Gigan, M. S. Kim, C. Brukner, J. Eisert, and M. Aspelmeyer, Phys. Rev. Lett. 99, 250401 (2007).

[4] W. Marshall, C. Simon, R. Penrose, and D. Bouwmeester, Phys. Rev. Lett. 91, 130401 (2003).
[5] A. Ferreira, A. Guerreiro, and V. Vedral, Phys. Rev. Lett. 96, 060407 (2006).

[6] S. Pirandola, D. Vitali, P. Tombesi, and S. Lloyd, Phys. Rev. Lett. 97, 150403 (2006).

[7] D. Vitali, S. Gigan, A. Ferreira, H. R. Bohm, P. Tombesi, A. Guerreiro, V. Vedral, A. Zeilinger, and M. Aspelmeyer, Phys. Rev. Lett. 98, 030405 (2007).

[8] K. C. Schwab and M. L. Roukes, Phys. Today 58, 36 (2005); M. Poot and H. S. J. van der Zant, Phys. Rep., in press (2011), e-print arXiv:1106.2060. 
[9] P. Rabl, A. Shnirman, and P. Zoller, Phys. Rev. B 70, 205304 (2004); M. D. LaHaye, O. Buu, B. Camarota, and K. C. Schwab, Science 304, 74 (2004); A. Naik et al., Nature (London) 443, 193 (2006); S. Pugnetti, Y. M. Blanter, and R. Fazio, Europhys. Lett. 90, 48007 (2010).

[10] G. Vacanti et al., submitted for publication (2010).

[11] M. V. Berry, Proc. Roy. Soc. A 329, 45 (1984).

[12] Y. Aharonov and J. Anandan, Phys. Rev. Lett. 58, 1593 (1987).

[13] A. Carollo, I. Fuentes-Guridi, M. F. Santos, and V. Vedral, Phys. Rev. Lett. 90, 160402 (2003); 92, 020402 (2004).

[14] D. Leibfried et al., Nature (London) 422, 412 (2003).

[15] G. J. Milburn, S. Scheneider, and D. F. V. James, Fortschr. Phys. 48, 801 (2000).

[16] $\operatorname{Im}\left(\oint \zeta^{*} d \zeta\right)=\oint x d y-y d x=\int_{\sigma} d x d y=\mathcal{A}$, where we have taken $\zeta=x+i y$.

[17] The same result, i.e., an overall phase $\vartheta_{\text {tot }}$ equal to $\mathcal{A}$ for an arbitrary closed loop, is obtained using Anandan's rule, $\vartheta_{\text {tot }}=$ $\theta_{D}+\theta_{G}$, with $\theta_{D}=-1 / \hbar \int_{0}^{\tau}\langle\psi(t) H(t) \psi(t)\rangle d t$ the dynamical phase, $\theta_{G}=i \int_{0}^{\tau}\left\langle\psi(t) \partial_{t} \psi(t)\right\rangle d t$ the geometric one [12], and $\tau$ and $\tau_{0}$ the initial and final times of the evolution.

[18] L. M. Arévalo-Aguilar and H. Moya-Cessa, Quantum Semiclass. Opt. 10, 671 (1998)

[19] E. Sjoqvist, A. K. Pati, A. Ekert, J. S. Anandan, M. Ericsson, D. K. L. Oi, and V. Vedral, Phys. Rev. Lett. 85, 2845 (2000).

[20] H. F. Trotter, Proc. Am. Math. Soc. 10, 545 (1959); M. Suzuki, Commun. Math. Phys. 51, 183 (1976).

[21] For the Hamiltonian considered here, the chosen approach is an excellent approximation of the exact dynamics of the system. Introducing the superoperator $\hat{\mathcal{H}} \rho=-i[\hat{H}, \rho]$, we write the formal solution of the open-system dynamics as $\rho(t)=$
$e^{\hat{\mathcal{H}} t+\hat{\mathcal{L}} t} \rho_{0}$. Upon explicit calculation, it is straightforward to show that, as $\delta t \rightarrow 0,\left[\hat{\mathcal{D}}_{\delta t}, \hat{\mathcal{U}}_{\delta t}^{\varphi}\right]=0$, so that $\rho(N \delta t) \rightarrow \rho(t)$ in this limit.

[22] H. Jeong, Phys. Rev. A 72, 034305 (2005).

[23] S. J. D. Phoenix, Phys. Rev. A 41, 5132 (1990).

[24] This condition can be met by taking $T_{1,2}$ such that $e^{-\gamma T_{1}}=$ $2-e^{\gamma T_{2}}$.

[25] The complete expression of $\tilde{\Gamma}\left(\gamma, T_{1}, T_{2}\right)$ is $\tilde{\Gamma}\left(\gamma, T_{1}, T_{2}\right)=\gamma \tau+$ $\frac{1}{4}\left(1-e^{-\gamma T_{1}}\right)^{2}\left(1-e^{-2 \gamma T_{2}}\right)+\frac{1}{2}\left(2-e^{-2 \gamma T_{1}}-e^{-2 \gamma T_{2}}\right)-2(2-$ $\left.e^{-\gamma T_{1}}-e^{-\gamma T_{2}}\right)-\left(1-e^{-\gamma T_{1}}\right)\left[\left(1-e^{-\gamma T_{2}}\right)-\frac{1}{2}\left(1-e^{-2 \gamma T_{2}}\right)\right]$. Truncating the expansion of the exponential function to the second order in $\gamma T_{j} \ll 1$, the only non-null terms are those $\propto \gamma^{3}$.

[26] I. Wilson-Rae, P. Zoller, and A. Imamoglu, Phys. Rev. Lett. 92, 075507 (2004).

[27] M. Wallquist, K. Hammerer, P. Zoller, C. Genes, M. Ludwig, F. Marquardt, P. Treutlein, J. Ye, and H. J. Kimble, Phys. Rev. A 81, 023816 (2010); K. Hammerer, M. Wallquist, C. Genes, M. Ludwig, F. Marquardt, P. Treutlein, P. Zoller, J. Ye, and H. J. Kimble, Phys. Rev. Lett. 103, 063005 (2009).

[28] D. E. Chang, C. A. Regal, S. B. Papp, D. J. Wilson, J. Ye, O. Painter, H. J. Kimble, and P. Zoller, Proc. Natl. Acad. Sci. USA 107, 1005 (2010); O. Romero-Isart, M. L. Juan, R. Quidant, and J. Ignacio Cirac, New J. Phys. 12, 033015 (2010); M. Paternostro, G. De Chiara, and G. M. Palma, Phys. Rev. Lett. 104, 243602 (2010); C. Genes, D. Vitali, and P. Tombesi, Phys. Rev. A 77, 050307(R) (2008); G. De Chiara, M. Paternostro, and G. M. Palma, ibid. 83, 052324 (2011).

[29] P. Verlot, A. Tavernarakis, T. Briant, P. F. Cohadon, and A. Heidmann, Phys. Rev. Lett. 104, 133602 (2010).

[30] M. Pechal et al., e-print arXiv:1109.1157 (2011). 